\title{
Exergaming for balance training of elderly: state of the art and future developments
}

\author{
Mike van Diest ${ }^{1,2^{*}}$, Claudine JC Lamoth², Jan Stegenga ${ }^{1}$, Gijsbertus J Verkerke ${ }^{3,4}$ and Klaas Postema ${ }^{3}$
}

\begin{abstract}
Fall injuries are responsible for physical dysfunction, significant disability, and loss of independence among elderly. Poor postural control is one of the major risk factors for falling but can be trained in fall prevention programs. These however suffer from low therapy adherence, particularly if prevention is the goal. To provide a fun and motivating training environment for elderly, exercise games, or exergames, have been studied as balance training tools in the past years. The present paper reviews the effects of exergame training programs on postural control of elderly reported so far. Additionally we aim to provide an in-depth discussion of technologies and outcome measures utilized in exergame studies. Thirteen papers were included in the analysis. Most of the reviewed studies reported positive results with respect to improvements in balance ability after a training period, yet few reached significant levels. Outcome measures for quantification of postural control are under continuous dispute and no gold standard is present. Clinical measures used in the studies reviewed are well validated yet only give a global indication of balance ability. Instrumented measures were unable to detect small changes in balance ability as they are mainly based on calculating summary statistics, thereby ignoring the time-varying structure of the signals. Both methods only allow for measuring balance after the exergame intervention program. Current developments in sensor technology allow for accurate registration of movements and rapid analysis of signals. We propose to quantify the time-varying structure of postural control during gameplay using low-cost sensor systems. Continuous monitoring of balance ability leaves the user unaware of the measurements and allows for generating user-specific exergame training programs and feedback, both during one game and in timeframes of weeks or months. This approach is unique and unlocks the as of yet untapped potential of exergames as balance training tools for community dwelling elderly.
\end{abstract}

Keywords: Postural control, Sensors, Exergames, Balance training, Elderly, Fall prevention

\section{Introduction}

More than one third of the community-dwelling individuals aged 65 and older falls at least once per year [1,2]. Approximately $5-20 \%$ of the falls have serious consequences including major head trauma, major lacerations, or fracture and may lead to immobility or death $[1,3]$. The severity of the consequences of falls increases with ageing and the number of injuries and injurious falls strongly predict placement in a nursing home $[4,5]$.

Impaired postural control with muscle weakness is an important predictor of falls within the elderly population

\footnotetext{
* Correspondence: mikevandiest@incas3.eu

${ }^{1}$ INCAS ${ }^{3}$, Dr. Nassaulaan 9, 9401 HJ Assen, The Netherlands

${ }^{2}$ Center for Human Movement Sciences, University of Groningen, University

Medical Center Groningen, Hanzeplein 1, 9713 GZ Groningen, The

Netherlands

Full list of author information is available at the end of the article
}

[6,7]. Postural control is defined in this review as the ability to maintain, achieve, or restore a state of balance during any posture or activity [8]. Ageing has a detrimental effect on postural control either due to a specific pathology affecting a particular component of the sensory, motor and central processing systems, and/or as a consequence of a more general age-related deterioration of sensory and neuromuscular control mechanisms $[9,10]$. Appropriate control of posture underlies many motor skills and is an absolute pre-requisite for activities of daily living. Postural control entails accurately timed vestibular, visual, proprioceptive and somatosensory inputs for adaptive strategies for orientation and balance [9]. For the simple act of reaching for a cup, one must permanently monitor all the afore-listed inputs and perform subtle adjustments to coordinate movement. 
Integration of sensory inputs occurs without conscious attention, so people are routinely able to perform two tasks simultaneously, like talking and walking [11]. However, it has been shown that the control of posture, even in young adults, can suffer from the concurrent execution of dual tasks, thus placing a heavy load on working memory capacity $[12,13]$. The age-related loss of visual, proprioceptive, and vestibular sensitivity demands more attention for maintaining postural stability during standing and walking [14-16]. In that case, responses to dual tasking could destabilize motor activity $[17,18]$. Moreover, ageing is not only associated with impaired movement dynamics but also with a deterioration of cognitive processes involving working memory. One may thus pinpoint two major reasons for a reduced postural control during execution of a concurrent task: A greater need for conscious attention to maintain good postural control due to impaired sensory and motor system function and, by the same token, a reduced attentional and working memory capacity.

Many interventions for fall risk and fall rate reduction have been studied in the past years. Components of intervention programs include strength, balance and gait training, improving ambulation with improved footwear, walking aids, fall training, medication review, vision tests, fall sensors, home risk assessment and hip protectors $[19,20]$. Key components of fall prevention training programs for community dwelling elderly include balance, muscle-strength, flexibility and endurance [21]. The greatest effects on fall rate were seen in programs that included a combination of a high dose of exercise $(>50$ hours over the trial period) and challenging balance exercises [22].

A drawback of conventional exercise programs is however, that they suffer from low adherence, particularly if prevention is the goal as those programs usually start after one or more falls with serious consequences [22-24]. Exergames (exercise + gaming) appear promising for home-based balance and strength training for healthy elderly. Exergaming devices have several advantages compared to conventional exercises; exergaming can motivate people to practice and by performing dual tasks users can train both cognitive and motor skills. Additionally the focus of attention is not on the movements itself, but on the outcome of the movements in the game. This is important as in daily life one is also focused on the outcome of movements rather than consciously aiming to maintain balance [24-26].

Gaming technologies have become increasingly affordable and accessible over the past few years and exergames have therefore gained much interest in the field of mobility training for healthy individuals as well as for specific pathologies including stroke, SCI and cerebral palsy. Plow et al. reported in a scoping review of exergaming for adults with systemic disabling conditions that most of the studies reviewed showed potential to improve functional ability in the target population but that the field is still in its infancy and that there are few controlled trials [27]. The current review aims to provide an overview of the exergames that have been used for the specific purpose of training balance in the elderly population. Commercial systems developed specifically for training balance in elderly are rare [28] and most studies rely on off-theshelf systems with commercial software not specifically developed for elderly or custom software developed for experimental balance training purposes. Although a rapidly growing number of studies are published about exergames, well-controlled studies are still rare. Therefore the present paper will not provide a systematic review of the literature, but provide an in-depth description and discussion of results obtained so far from existing commercial-of-the-shelf systems and custom-designed exergames to train balance. More specifically, 1) technologies and 2) outcome measures used and 3) the effects of the exergame interventions in the elderly population will be discussed. Finally, based on these results we will provide a conceptual framework of the possibilities of exergames as a balance training tool for elderly.

\section{Search criteria}

Pubmed and Web of Science were searched to provide an overview of the technologies, outcome measures used, and the effects of exergames on balance ability. The keywords used in the search were: [serious games OR exergames OR virtual reality OR computer assisted OR video] AND [balance OR postural stability OR postural control] AND [training OR exercise OR fall prevention]. A selection was made based on the following inclusion criteria: The study should evaluate a commercial of-the-shelf system or evaluate or propose a custom exergame or VRtool with game elements used for training balance in elderly or adults. Additional studies were identified by scanning reference lists. It was decided to exclude the studies involving pathological states including Cerebral Palsy (CP), Stroke, Acquired brain injury (ABI) and spinal cord injury (SCI) as these users show little similarity with community dwelling elderly, thereby including 13 studies.

\section{Exergaming devices}

Exergame devices are controlled using a broad variety of sensor systems and, depending on the source of input, different algorithms are needed for game control and feedback. The most widely used sensors in exergame input devices include accelerometers, gyroscopes, infrared (IR) and RGB optical sensors/cameras and pressure sensors [29-34].

\section{Inertial sensors}

Inertial sensors encompassing accelerometers and gyroscopes are positioning sensors that measure accelerations 
and angular velocity respectively. A well-known commercial off-the-shelf game system that uses inertial sensors is the Nintendo Wii (Nintendo, Kyoto, Japan), which was introduced by Nintendo in 2006. Wii gameplay is controlled by the players' movements, measured with a wireless hand-held controller, a Wii remote, in which a three-axis accelerometer and a single- and dual-axis gyroscope are embedded. By fusing the sensor data from the gyroscopes and the accelerometers, the Wii Remote can measure changes in direction, speed and acceleration with a sensitivity of $\pm 1 \%$ [35]. Inertial sensors measure specific force and angular rate without an external reference. Consequently, errors build up quickly over time and the precise position of the device is difficult to deduce from these signals. The Wii remote therefore has an additional optical sensor on the controller that measures the position of a sensor bar, mounted on the television, which emits 2 IR light signals. The distance between the lights and the relative angle between the IR lights provide information about the position of the controller. Using these sensors the Wii controller is able to measure both rapid (using inertial sensing) and slow (using the optical sensor) movements.

Inertial sensors have also been used in wobble boards for training balance (see Table 1) [24,25,33]. These boards consist of an instable plate, which causes the user, while standing on the plate, to wobble, thereby controlling the game, for instance a ball in a maze [33], by shifting his weight in ML and AP directions. The movements are measured using a single orientation tracker (Xsens MTx Motion Tracker, Xsens Technologies, The Netherlands) which consists of three gyroscopes [36]. For global reference the MTx measures the direction of gravity and the magnetic north. The accuracy of the device is $<1 \mathrm{deg}$, the update rate of calibrated sensor data: $512 \mathrm{MHz}$.

\section{Pressure sensors}

Pressure sensors are also used widely as a game input device [23,29-31,37-45]. For instance, the Wii Balance Board (WBB) consists of a board $(51 \times 31 \mathrm{~cm})$ with 4 force transducers thereby allowing for calculation of the users center of pressure (COP) used for controlling games. Typical game tasks include shifting weight, taking poses or stepping on and off the WBB in the context of yoga or aerobics [42]. Comparable systems are a pressure mat [23] or panels with pressure sensors used for dancing games $[30,31]$.

\section{Camera systems}

Inertial and pressure sensors hold the limitation that the user is in direct contact with a controller. Alternatively, camera systems provide the possibility to play games without holding or wearing input devices [38,40,46-48]. The Sony PlayStation Eyetoy (Sony, Park Ridge, New Jersey, USA) for instance, uses a color video camera with a software package that enables gesture recognition to play games. Games are controlled by the movements of the user itself, rather than via a controller that is held by the user. The Eyetoy has been used predominantly for (upper extremity) rehabilitation purposes [32,46-48]. A disadvantage of this system is that the camera system does not provide the accuracy necessary for playing faster games or taking high resolution measurements. Commercially available webcams are also being used to control exergames [40].

A recent development is the field of exergames is the XBOX 360 Kinect (Microsoft corp., Redmond, WA). Like the Eyetoy it uses gesture recognition rather than a hand-held game controller. The system captures depth and color information and generates a point cloud of colored dots. The software is able to calculate the 3D position of the dots, thereby creating a 3D image of the environment. Algorithms analyze the sensor data and calculate the position of the user's body parts thereby allowing tracking of the users' movements, even when a body part is occluded [49]. Like the Eyetoy the Kinect does not allow for measurements with a resolution and sample frequency comparable to high-end camera systems. Although several studies are being conducted, no publications relating to balance training have been published on the cutoff date for this paper.

\section{Outcome measures: internal and external}

A broad variety of outcome measures for quantifying the effects of exergame interventions are reported in literature. Two different methods to assess performance can be discerned 1) during gameplay and 2) outside the game environment, in the present paper referred to as internal and external outcome parameters, respectively. Internal outcome measures are generated using an instrumented measurement tool and algorithms that convert the sensor data automatically into the outcome measure. The range of movement of the COP can for instance be measured using a pressure mat during a weight shifting game task [23]. External outcome measures are administered after gameplay. Examples include administering the Berg Balance Scale or sway variability during standstill after an intervention period [33]. Contrary to external outcome measures, internal outcome measures can provide the user with direct feedback during gameplay.

In several exergame studies the COP is used to control the game yet these measurements are not considered internal or external outcome measures as they do not quantify balance ability but only are used to play the game [38,39]. Table 1 provides an overview of outcome measures used in exergame studies, along with the technologies used to measure these parameters and the study results. 
Table 1 Overview technologies and results of studies evaluating exergames for balance training in elderly and adults

\begin{tabular}{|c|c|c|c|c|c|c|c|}
\hline & \multirow[t]{2}{*}{$\begin{array}{l}\text { Sample characteristics } \\
\text { \& Study type }\end{array}$} & \multirow[t]{2}{*}{$\begin{array}{l}\text { Technologies } \\
\text { used }\end{array}$} & \multirow[t]{2}{*}{ Game elements and tasks } & \multirow[t]{2}{*}{$\begin{array}{l}\text { Intervention } \\
\text { design }\end{array}$} & $\begin{array}{l}\text { Internal outcome } \\
\text { measures }\end{array}$ & \multirow[t]{2}{*}{$\begin{array}{l}\text { External outcome } \\
\text { measures }\end{array}$} & \multirow[t]{2}{*}{ Results } \\
\hline & & & & & $\begin{array}{l}\text { Feedback to } \\
\text { user (FB) }\end{array}$ & & \\
\hline Studenski [30] & $\begin{array}{l}\text { E: Healthy elderly }(\mathrm{N}=36) \\
\text { Age: } 80.1 \pm 5.4 \\
\text { Study type: UCT }\end{array}$ & $\begin{array}{l}\text { Dance pad } \\
\text { containing } \\
\text { four panels }\end{array}$ & $\begin{array}{l}\text { Dance game (DancetownTM) } \\
\text { controlled by stepping movements } \\
\text { in forward, backward left and right } \\
\text { directions. }\end{array}$ & $\begin{array}{l}24 \times 30 \text { min } \\
\text { sessions over } \\
3 \text { months. } \\
\text { Total }=720 \mathrm{~min} .\end{array}$ & $\begin{array}{l}\text { Not used } \\
\text { FB user: Game } \\
\text { performance }\end{array}$ & $\begin{array}{l}\text { - BMI } \\
\text { - blood pressure } \\
\text { - Pulse } \\
\text { - SPPB } \\
\text { - NWT } \\
\text { - Digit symbol } \\
\text { substitution test } \\
\text { - SF36 PMC } \\
\text { - ABC scale }\end{array}$ & $\begin{array}{l}\text { SF } 36 \text { PMC, NWT and ABC scale } \\
\text { improved }(p<0.05) \\
\text { No significant effects on other } \\
\text { outcome measures }\end{array}$ \\
\hline Smith [31] & $\begin{array}{l}\text { E 1: Healthy elderly }(\mathrm{N}=26) \\
\text { Age: } 78.9 \pm N R \\
\mathrm{E} 2 \text { and } \mathrm{E} \text { 3: Healthy elderly } \\
(\mathrm{N}=20) \\
\text { Age: } 79.6 \pm \mathrm{NR} \\
\text { C: Young adults }(\mathrm{N}=20) \\
\text { Age: } 28.4 \pm \mathrm{NR} \\
\text { Study type: } C T\end{array}$ & $\begin{array}{l}\text { Dance mat } \\
\text { containing } \\
\text { four panels }\end{array}$ & $\begin{array}{l}\text { E 1: Stepping to the left, right, left } \\
\text { forward or right forward on panels } \\
\text { corresponding to arrows shown on } \\
\text { a display. Arrow drift speed varied. } \\
\text { E 2: Same game with constant } \\
\text { arrow speed. } \\
\text { E 3: Manipulated arrow speed and } \\
\text { appearance }\end{array}$ & $\begin{array}{l}\text { E 1: } 4 \text { min } \\
\text { E } 2 \text { and E } 3: 5 x \\
4 \text { min trials }<1 \text { hr. } \\
\text { Total Studies } 2 \text { and } \\
3=80 \text { min. }\end{array}$ & $\begin{array}{l}\text {-Step timing } \\
\text {-\% missed targets } \\
\text { FB user: not } \\
\text { reported }\end{array}$ & Not used & $\begin{array}{l}\text { Step timing improved }(p<0.05) \\
\text { after multiple trials \& group } \\
\text { effect; young outperformed } \\
\text { elderly }(p<0.05) \text {. } \\
\% \text { missed targets decreased after } \\
\text { multiple trials }(p<0.05) \& \text { group } \\
\text { effect; young adults } \\
\text { outperformed elderly }(p<0.05) \text {. }\end{array}$ \\
\hline Kosse [33] $]^{1}$ & $\begin{array}{l}\text { E: Healthy elderly }(\mathrm{N}=9) \\
\text { Age: } 77 \pm 5.0 \\
\text { Study type: ITS }\end{array}$ & Wobble board & $\begin{array}{l}\text { Moving a ball through a maze } \\
\text { using weight shifts without lifting } \\
\text { the feet }\end{array}$ & $\begin{array}{l}18 \times 20 \text { min. over } \\
6 \text { weeks } \\
\text { Total }=360 \text { min. }\end{array}$ & $\begin{array}{l}\text { Not used } \\
\text { FB user: Game } \\
\text { performance }\end{array}$ & $\begin{array}{l}\text { - BBS } \\
\text { - FOE } \\
\text { - TS EO and EC } \\
\text { - OLS EO and EC }\end{array}$ & $\begin{array}{l}\text { BBS and FOE improved }(p<0.01) \\
\text { TS and OLS EC \& EO did not } \\
\text { improve }\end{array}$ \\
\hline Fitzgerald [24] & $\begin{array}{l}\text { E: Healthy adults }(\mathrm{N}=11) \\
\text { Age: } 25.4 \pm 2.1 \\
\text { C: Healthy adults }(\mathrm{N}=11) \\
\text { Age: } 26.9 \pm 3.2 \\
\text { Study type: } \mathrm{RCT}\end{array}$ & Wobble board & $\begin{array}{l}\text { E: Controlling a ball using weight } \\
\text { shifts without lifting the feet } \\
\text { C: Postural stability training on a } \\
\text { wobble board. }\end{array}$ & $\begin{array}{l}3 \times 15 \text { min. } \mathrm{p} / \mathrm{wk} \\
\text { for } 4 \text { weeks } \\
\text { Total = } 180 \mathrm{~min} .\end{array}$ & $\begin{array}{l}\text { Not used } \\
\text { FB user: Game } \\
\text { performance }\end{array}$ & $\begin{array}{l}\text { - DPSI } \\
\text { - SEBT } \\
\text { - Intrinsic motivation } \\
\text { inventory }\end{array}$ & $\begin{array}{l}\text { DPSI and SEBT: no group effects. } \\
\text { Intrinsic motivation: higher }(p< \\
0.01) \text { score on interest/ } \\
\text { enjoyment category }\end{array}$ \\
\hline${\text { Lamoth }[25]^{1}}^{1}$ & $\begin{array}{l}\text { E: Healthy elderly }(\mathrm{N}=9) \\
\text { Age: } 77 \pm 5.0 \\
\text { Study type: ITS }\end{array}$ & Wobble board & $\begin{array}{l}\text { Moving a ball through a maze } \\
\text { using weight shifts without lifting } \\
\text { feet. }\end{array}$ & $\begin{array}{l}18 \times 20 \text { min over } \\
6 \text { weeks } \\
\text { Total }=360 \mathrm{~min} .\end{array}$ & $\begin{array}{l}\text { Not used } \\
\text { FB user: Game } \\
\text { performance }\end{array}$ & $\begin{array}{l}\text { - DT } \\
\text { - Standing FP\& TS: trunk } \\
\text { acceleration patterns } \\
\text { in AP and ML: } \\
\text { variability, regularity, } \\
\text { smoothness. }\end{array}$ & $\begin{array}{l}\text { Performance on DT improved } \\
(p<0.05) \text {, Postural control indexed } \\
\text { by variability, regularity and } \\
\text { smoothness improved }(P<0.05)\end{array}$ \\
\hline Betker [23] & $\begin{array}{l}\text { Healthy Adults }(N=8) \\
\text { Neurological patients } \\
(N=7) \\
\text { Age: } 15-72 \\
\text { Study type: } C T\end{array}$ & $\begin{array}{l}\text { Pressure mat, } \\
53 \times 53 \mathrm{~cm}, \\
256 \text { pressure } \\
\text { sensors. }\end{array}$ & $\begin{array}{l}\text { Weight shifts in } \mathrm{AP} \text { and } \mathrm{ML} \\
\text { direction with cognitive tasks. }\end{array}$ & $\begin{array}{l}3 \times 10 \mathrm{~min} \\
\text { Total }=30 \mathrm{~min}\end{array}$ & $\begin{array}{l}\text { During gameplay: } \\
\text { game } \\
\text { performance, COP } \\
\text { position } \\
\text { After gameplay } \\
\text { total ROM ML \& } \\
\text { AP directions. }\end{array}$ & $\begin{array}{l}\text { - Questionnaire: } \\
9 \text { questions about } \\
\text { enjoyment, motivation } \\
\text { to exercise, game } \\
\text { difficulty }\end{array}$ & $\begin{array}{l}\text { Internal outcome measures: NR } \\
\text { Questionnaire: Games are } \\
\text { challenging, attractive and more } \\
\text { appealing than traditional } \\
\text { exercises. }\end{array}$ \\
\hline
\end{tabular}


Table 1 Overview technologies and results of studies evaluating exergames for balance training in elderly and adults (Continued)

\begin{tabular}{|c|c|c|c|c|c|c|c|}
\hline Bisson [38] & $\begin{array}{l}\text { E 1: Healthy elderly }(\mathrm{N}=12) \\
\text { Age: } 74.4 \pm 3.65 \\
\text { E 2: Healthy elderly }(\mathrm{N}=12) \\
\text { Age: } 74.4 \pm 4.92 \\
\text { Study type: ITS }\end{array}$ & $\begin{array}{l}\text { Camera tracks } \\
\text { red gloves, } \\
\text { player } \\
\text { projected in } \\
\text { Virtual } \\
\text { Environment. } \\
\text { Force platform } \\
\text { measures COP. }\end{array}$ & $\begin{array}{l}\text { E 1: Moving cursor using weight } \\
\text { shifts } \\
\text { E 2: Catch 'Falling' balls by } \\
\text { reaching the arms without lifting } \\
\text { feet }\end{array}$ & $\begin{array}{l}2 \times 30 \text { min. } \mathrm{p} / \mathrm{wk} \text { for } \\
10 \text { weeks } \\
\text { Total }=600 \\
\text { minutes }\end{array}$ & $\begin{array}{l}\text { Not used } \\
\text { FB user: Game } \\
\text { performance }\end{array}$ & $\begin{array}{l}\text { - Sway variability AP } \\
\text { and ML } \\
\text { - CB\&M } \\
\text { - RT }\end{array}$ & $\begin{array}{l}\text { Sway variability: no significant } \\
\text { group effect. } \\
\text { CB\&M and RT: both groups } \\
\text { improved }(P<0.05)\end{array}$ \\
\hline Lange [40] & $\begin{array}{l}\text { E: Healthy adults }(\mathrm{N}=7) \\
\text { Age: } 16-43 \\
\text { Study type: UCT }\end{array}$ & $\begin{array}{l}\text { Webcams, LED } \\
\text { markers, } \\
\text { custom step- } \\
\text { based game. }\end{array}$ & $\begin{array}{l}\text { Dance and step-based exercises in } \\
\text { forward, backward, left, right and } \\
\text { diagonal directions }\end{array}$ & Not specified & $\begin{array}{l}\text { Not used } \\
\text { FB user: Not } \\
\text { reported }\end{array}$ & $\begin{array}{l}\text { - Semi-structured } \\
\text { interviews about } \\
\text { game experience }\end{array}$ & $\begin{array}{l}\text { All participants reported enjoying } \\
\text { the experience }\end{array}$ \\
\hline Nitz [41] & $\begin{array}{l}\text { E: Healthy adults }(\mathrm{N}=8) \\
\text { Age: } 46.6 \pm 9.9 \\
\text { Study type: UCT }\end{array}$ & $\begin{array}{l}\text { Wii balance } \\
\text { board }\end{array}$ & $\begin{array}{l}\text { WiFit: Yoga, balance, aerobic and } \\
\text { strength pre-programmed } \\
\text { activities. }\end{array}$ & $\begin{array}{l}2 \times 30 \mathrm{~min} p / \mathrm{wk} \text { for } \\
10 \text { weeks. } \\
\text { Total }=600 \mathrm{~min} .\end{array}$ & $\begin{array}{l}\text { Not used } \\
\text { FB user: Game } \\
\text { performance }\end{array}$ & $\begin{array}{l}\text { - 6MWT } \\
\text { - TUG } \\
\text { - TUGcognitive } \\
\text { - Step test } \\
\text { - BS (foam, EC) } \\
\text { - OLS (EO) } \\
\text { - LOS } \\
\text { - RT } \\
\text { - Ankle dorsiflexion } \\
\text { - LLS } \\
\text { - Body weight }\end{array}$ & $\begin{array}{l}\text { OLS for both limbs and lower } \\
\text { limb strength improved }(P<0.05) \\
\text { Other outcome measures: no } \\
\text { significant improvement. }\end{array}$ \\
\hline Williams [42] & $\begin{array}{l}\text { E: Elderly with increased } \\
\text { fall risk }(N=15) \\
\text { Age: } 76.8 \pm 5.2 \\
\text { C: Elderly with increased } \\
\text { fall risk }(N=6) \\
\text { Age: } 76.5 \pm 4.8 \\
\text { Study type: } C T\end{array}$ & $\begin{array}{l}\text { Nintendo Wii, } \\
\text { Wii balance } \\
\text { board, walking } \\
\text { frame. }\end{array}$ & $\begin{array}{l}\text { E: WiiFit, balance and aerobic } \\
\text { exercises controlled using stepping } \\
\text { on the board, shifting weight and } \\
\text { performing poses. } \\
\text { C: Standard care exercise/ } \\
\text { education programme }\end{array}$ & $\begin{array}{l}\text { E: } 2 \text { game sessions } \\
\text { per week for } \\
12 \text { weeks. } \\
\text { Total }=\text { Not reported } \\
\text { C: } 12 \text { week } \\
\text { exercise/education } \\
\text { programme }\end{array}$ & $\begin{array}{l}\text { Wii Age; number } \\
\text { calculated by the } \\
\text { Nintendo Wii based } \\
\text { on game results. } \\
\text { FB user: Game } \\
\text { performance, Wii } \\
\text { Age. }\end{array}$ & $\begin{array}{l}\text { - BBS } \\
\text { - TBA } \\
\text { - FES-I }\end{array}$ & $\begin{array}{l}\text { No group effect on BBS and TBA. } \\
\text { Control group improved on FES-I } \\
(P<0.05) \text {. Game group improved } \\
\text { on Wii-age }(P<0.05)\end{array}$ \\
\hline Young [43] & $\begin{array}{l}\text { E: Healthy elderly }(\mathrm{N}=6) \\
\text { Age: } 84.1 \pm 5.1 \\
\text { Study type: ITS }\end{array}$ & $\begin{array}{l}\text { Wii balance } \\
\text { board, custom } \\
\text { games. }\end{array}$ & $\begin{array}{l}\text { Catching apples and popping } \\
\text { balloons using COP shifts without } \\
\text { lifting feet. }\end{array}$ & $\begin{array}{l}10 \times 20 \text { min. over } \\
4 \text { weeks } \\
\text { Total }=200 \mathrm{~min} .\end{array}$ & $\begin{array}{l}\text { Not used } \\
\text { FB user: Real-time } \\
\text { visual FB of } \\
\text { current COP } \\
\text { position }\end{array}$ & $\begin{array}{l}\text { - Sway variability AP } \\
\text { EO \& EC } \\
\text { - Sway variability ML } \\
\text { EO \& EC } \\
\text { - FES }\end{array}$ & $\begin{array}{l}\text { Sway variability AP EC improved } \\
\text { ( } p<0.05 \text { ) } \\
\text { Other sway measures and FES } \\
\text { showed trend towards } \\
\text { improvement. }\end{array}$ \\
\hline Heiden [44] & $\begin{array}{l}\text { E: Healthy elderly }(\mathrm{N}=9) \\
\text { C: Healthy elderly }(\mathrm{N}=6) \\
\text { Age : } 77 \pm \mathrm{NR} \\
\text { Study type: } \mathrm{CT}\end{array}$ & $\begin{array}{l}2 \text { Force plates } \\
(25 \times 10 \times 1.5 \mathrm{~cm})\end{array}$ & $\begin{array}{l}\text { E: Playing tennis game 'Pong' } \\
\text { controlled using weight shifts in } \\
\text { AP and ML directions and a } \\
\text { dynamic stepping routine + chair } \\
\text { exercise program. } \\
\text { C: Chair exercise program. }\end{array}$ & $\begin{array}{l}\text { E: } 2 \times(16 \times 60 \text { min. } \\
\text { chair exercises }+ \\
30 \text { min. exergaming }) \\
\text { p/wk for } 8 \text { weeks. } \\
\text { Total exercise }= \\
1440 \text { min. and } \\
\text { gaming }=480 \text { min. } \\
\text { C: } 16 \times 60 \text { min. over } \\
8 \text { weeks } \\
\text { Total }=960 \text { min }\end{array}$ & $\begin{array}{l}\text { Not used } \\
\text { FB user: Real time } \\
\text { visual FB of COP } \\
\text { movement }\end{array}$ & $\begin{array}{l}\text { - Postural sway AP } \\
\text { - Postural sway ML } \\
\text { - RT dual task } \\
\text { - CB\&M } \\
\text { - } 6 \mathrm{MWT}\end{array}$ & $\begin{array}{l}\text { RT during dual task and CB\&M } \\
\text { improved ( } p<0.05 \text { ) in } \\
\text { intervention group } \\
\text { Both groups improved on the } \\
6 \text { MWT ( } p<0.05 \text { ). }\end{array}$ \\
\hline
\end{tabular}


Table 1 Overview technologies and results of studies evaluating exergames for balance training in elderly and adults (Continued)

\begin{tabular}{|c|c|c|c|c|c|c|c|}
\hline Agmon [75] & $\begin{array}{l}\text { E: Balance impaired elderly } \\
(N=7) \\
\text { Age: } 84 \pm 5 \\
\text { Study type: ITS }\end{array}$ & $\begin{array}{l}\text { Nintendo Wii, } \\
\text { Wii balance } \\
\text { board }\end{array}$ & $\begin{array}{l}4 \text { balance games controlled using } \\
\text { weights shifts and stepping on the } \\
\text { balance board }\end{array}$ & $\begin{array}{l}3 \times 30 \text { min } \mathrm{p} / \mathrm{w} \text { for } \\
3 \text { months. } \\
\text { Total }=1170 \mathrm{~min}\end{array}$ & $\begin{array}{l}\text { Not used } \\
\text { FB user: Game } \\
\text { performance }\end{array}$ & $\begin{array}{l}\text { - BBS } \\
\text { - Timed 4-meter walk } \\
\text { test } \\
\text { - PACES }\end{array}$ & $\begin{array}{l}\text { BBS and walking speed improved } \\
(P<0.05) \text {. } \\
\text { No effect on PACES. }\end{array}$ \\
\hline
\end{tabular}

$\mathrm{E}=$ Experimental group; $\mathrm{C}=$ Control group; $\mathrm{N}=$ Number of participants; $\mathrm{RCT}=$ Randomized controlled trial; $\mathrm{UCT}=$ Uncontrolled trial; $\mathrm{CT}=$ Controlled trial; ITS $=$ Interrupted time series design. When no $\mathrm{p}$ values are presented in the table, they were not provided in the paper, ${ }^{1}=$ Same data set; $\mathrm{NR}=$ Not reported; $\mathrm{ROM}=$ Range of movement; $\mathrm{ML}=$ Medio-lateral; $\mathrm{AP}=\mathrm{Anterior}$-posterior; $\mathrm{COP}=\mathrm{Centre}$ of pressure; $\mathrm{SF} 36 \mathrm{PMC}=\mathrm{SF} 36$ physical \& mental component; NWT $=$ Narrow walk time; DPSI = Dynamic postural stability index; SEBT $=$ Star excursion balance test; FOE $=$ Figure of eight; EO $=$ Eyes open; EC $=$ Eyes closed; DT $=$ Dot task; RT =

Reaction tim HS = Lower limb strength TUG = Timed-up-and go; Rea parallel; $T S=$ Tandem stance; OLS = One-leg stance; BS = Bilateral stance; PACES = Physical activity enjoyment scale. 
Clinical balance and mobility tests like the Berg Balance Scale (BBS) [50] and Timed Up and Go (TUG) $[51,52]$ are abundantly used to quantify the effect of an exergame intervention on postural control in young and older adults $[29,33,41,42,45,46]$ and are considered external measures. Other clinical tests that are used as outcome measures in the reviewed exergame studies include the Brunel balance assessment (BBA) [53], Anterior reach tests (ART) [54], Timed stair test (TST) [55], Stepping test (ST) [56], 1 minute walking test (1MWT) [57], 10 meter walking test (10MWT) [58], 30 seconds sit-to-stand test (30SST) [59], Community Balance and Mobility Scale (CB\&M) [60], Star Excursion Balance Test (SEBT) [61], figure of eight-test [62], the tandem stance and one-leg stance $[29,33,38,63]$. All these tests are suggested to provide information about postural control during different standing and walking tasks.

In exergaming studies the use of external balance measures based on sensor data is slowly emerging, but the number of studies using instrumented quantification of postural control is still small [23-25,31,33,38,41-44]. Most of these studies assessed balance by quantifying sway variability in mediolateral (ML) and anteroposterior (AP) direction during quiet stance using forceplates, as is widely used to measure balance ability in young and older, healthy and pathologic individuals [38,41,43,44,64-67]. Postural control was also assessed using trunk acceleration time series where regularity, variability and smoothness of the trunk acceleration patterns in ML and AP direction were quantified using the root mean square, sample entropy and mean power frequency respectively [25,33]. Other instrumented external outcome parameters that are supposed to indirectly determine balance and were reported in the studies reviewed include measurement of the ankle dorsiflexion, Limits of Stability (LOS), quadriceps strength, tactile acuity and body weight [41] and dynamical postural stability index (DPSI) $[24,68]$, the latter being a method in which the subject jumps and has to stand still for 3 seconds on a forceplate after landing.

For measuring game enjoyment, motivation, perceived exertion, cognitive abilities, mental health, self-efficacy and balance confidence, questionnaires including custom made questionnaires regarding game enjoyment [23] and intrinsic motivation [24], semi-structured interviews [40], the digit symbol substitution test (DSST) [69], the SF 36 mental component summary [70], Fall Efficacy Scale [71], Falls Efficacy Scale international (FES-I) [72] and Activity Specific Balance Confidence (ABC) scale [73] are used respectively.

Internal outcome measures used in the studies reviewed include step timing and the percentage of missed targets [31] and the total movement range of COP in ML and AP direction [23], all measured using pressure mats. Several exergame studies evaluated the effect of Wii games or custom games that make use of the Wii balance board, but few studies use this board as a source for internal outcome measures $[42,43]$. The validity of the internal instrumented measures used in the studies reviewed is under debate as they are often game-specific and although sometimes correlated with established measures, they are often not validated using follow-up studies on fall risk. An increase in these measures can reflect both an improvement in balance ability as well as a training effect of the game. Of the studies reviewed, only Smith et al. validated their internal outcome measures [74].

\section{Effects of exergaming on balance ability in adults and elderly}

Of the 13 studies reviewed, 5 used a (randomized) controlled trial design $[23,24,31,42,44]$ and 8 did not use a control group [25,30,33,38,40,41,43,75]. The results are summarized in Table 1. Two studies aimed to improve balance in healthy adults and used external outcome measures, both clinical and instrumented, for balance assessment $[24,41]$. Significant effects were found in SEBT score in posterolateral and posteromedial direction after playing wobble board games controlled using weight shifts. No effects were found using the instrumented measure, the DPSI [24]. Playing WiiFit games using a WBB significantly improved sway variability during unilateral stance for both limbs and lower limb strength but no effects were found using clinical measures including 6MWT and TUG [41]. Assessment of game enjoyment, motivation to exercise and game experience in healthy adults was assessed using questionnaires and interviews and it was found that all participants enjoyed playing the exergames $[23,40]$.

The effects of exergaming on age-related impaired postural control in elderly was examined in 9 studies $[25,30,31,33,38,42-44,75]$. One study used internal, 7 studies external and 1 study used both internal and external measures to assess balance ability. Of the studies using external measures, 3 used clinical, 1 used instrumented and 4 both clinical and instrumented measures. The largest effects were observed for clinical tests assessing balance during walking and during several standing tasks; significant improvements over the intervention period were found in CB\&M score [38,44], narrow walk time, $\mathrm{ABC}$ scale [30], BBS [33,42,75], walking speed [75], 6MWT [44] and FOE [33]. Standing balance was assessed using summary statistics in 4 studies using various instrumented measures but few found significant improvements over the intervention period, except for the sway variability in AP direction in eyes closed condition $[33,38,43,44]$. Also reaction time improved significantly $[38,44]$. Internal balance measures were used by 2 studies; improvements in step timing and percentage missed targets were observed after five 4-minute trials. Additionally, it was found that young adults outperform older adults 
[31]. As is shown in Figures 1 and 2, older adults predominantly improved on measures with a dynamic component such as walking, reaching and performing standing tasks. The effects of exergame programs are not often found significant when instrumented parameters measuring postural sway during quiet stance are applied. Also significant differences between intervention and control groups are scarce. Of the 13 studies reviewed 10 reported improvements in at least one balance measure after an exergame intervention period. The tasks performed in these effective games included stepping and weight shifting tasks.

\section{Discussion}

The present paper aims to provide a description and discussion of technologies and outcome measures used in exergames for balance training, covering both commercial off-the-shelf and custom hardware and games.

Recent developments in the field of sensor technology unlock great opportunities for using exergames as balance training tools. The studies reviewed applied inertial, pressure, and optical sensors for controlling exergames. Inertial and pressure sensors were used mostly [24,25,30,33,41-44], but camera systems are also becoming more widely used $[38,40,46]$. Cameras and gesture recognition software are expected to increase in popularity for rehabilitation purposes as they are getting cheaper, accuracy is increasing and gesture recognition is improving; these are important conditions for use in the home environment [76]. Inertial sensors however also become smaller and more energy efficient, thereby making them less obtrusive during gameplay [77]. Developments in the field of energy scavenging might even make manual recharging of batteries of inertial sensors obsolete [78].
Additionally the developments in the field of wireless sensor networks enable faster transfer and fusion of data of multiple inertial sensors, thereby enabling high sample frequency and more accurate measurements.

The broad variety of sensors, outcome measures and study designs used in the exergame studies reviewed indicates that the field of balance training is young and rapidly developing. Of the 13 studies included, 5 used a controlled trial design $[23,24,31,42,44]$. Of the studies that used an interrupted time series design, few included adequate number of participants and/or a control group. Moreover, studies are hard to compare as they use a broad variety of balance outcome measures, making it incongruous to draw conclusions regarding the effects of interventions.

In this paper internal and external outcome parameters were discerned and external parameters were classified as either clinical or instrumented. Significant improvements in balance ability were observed in studies assessing balance during walking or performing tasks using clinical tests $[24,30,33,38,42,44,75]$. The advantage of clinical balance tests is they are validated and easy to administer. A disadvantage however is that most of them only provide a global assessment of balance performance, and suffer from limitations including ceiling effects and limited precision to detect small changes in balance ability, which makes it onerous to estimate clinical relevance [50,52,60,72,79]. For instance, if a Berg Balance Score is higher or walking speed is faster, the cause of the change remains unclear. The exact aspects of postural control that are influenced by the intervention thus remain unclear.

Instrumented outcome measures can provide detailed information about changes in postural control. Only a

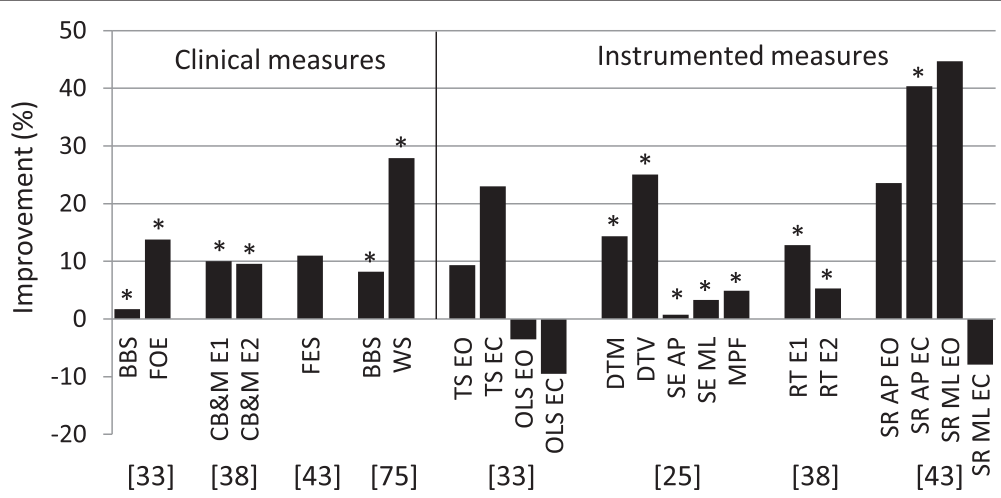

Figure 1 Summary of results of uncontrolled studies evaluating training effect of exergames on balance ability of elderly. The vertical axis represents the percentage improvement between pre- and post-intervention on the outcome measure provided on the horizontal axis. The bars on the left side of the solid vertical line indicate clinical measures, the bars on the right side instrumented measures. BBS $=$ Berg Balance Scale, FOE = Figure of Eight, $C B \& M=$ Community Balance and Mobility scale, E1 = experimental group 1, E2 = experimental group 2, FES = Fall Efficacy Scale, WS = Walking speed, TS = tandem stance time, OLS = one-leg stance time (both TS and OLS are measured in seconds), DTM = Dot gaming Task Mean, DTV = Dot gaming Task Variability, SR = Sway Root mean square values, EO = eyes open, EC = eyes closed, SE = sample entropy, $\mathrm{AP}=$ antero-posterior direction, $\mathrm{ML}=$ medio-lateral direction, $\mathrm{MPF}=$ mean power frequency, $\mathrm{RT}=$ reaction time, $\mathrm{SR}=$ Sway Root mean square values, * indicates a significance of $\mathrm{P}<0.05$. 


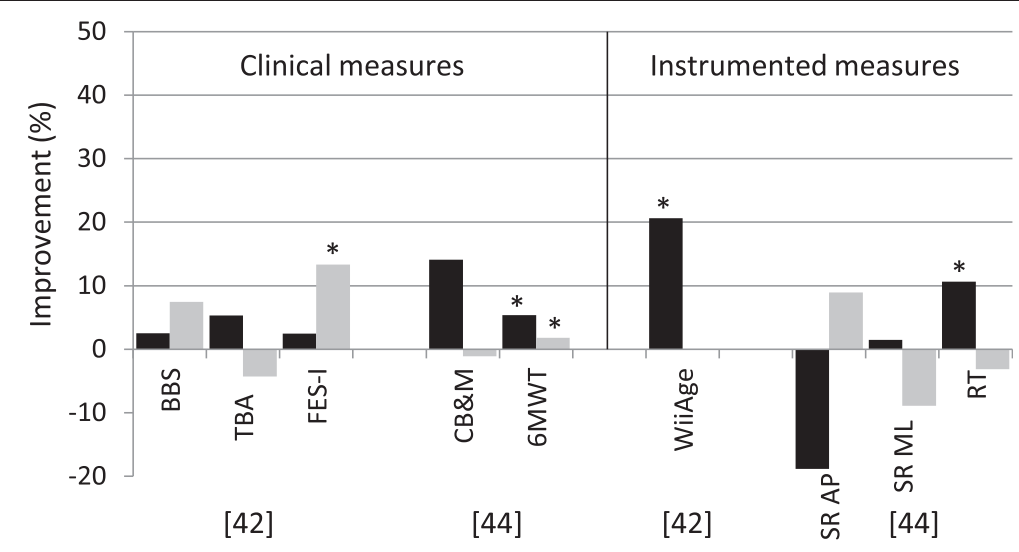

Figure 2 Summary of results of controlled studies evaluating training effect of exergames on balance ability of elderly. The vertical axis represents the percentage improvement between pre- and post-intervention on the outcome measure provided on the horizontal axis. Dark bars indicate the experimental group, light bars the control group. Note that [42] found an improvement in the control group where the experimental group did not improve. The bars on the left side of the solid vertical line indicate clinical measures, the bars on the right side instrumented measures. BBS = Berg Balance Scale, TBA = Tinetti Balance Assessment, FES-I = Fall Efficacy Scale International, CB\&M = Community Balance and Mobility scale, $6 \mathrm{MWT}=6$ Minute Walk Test, $\mathrm{SR}=$ Sway Root mean square values, $\mathrm{AP}=$ antero-posterior direction, $\mathrm{ML}=$ medio-lateral direction, RT $=$ reaction time. ${ }^{*}$ indicates a significance of $\mathrm{P}<0.05$.

limited number of the studies reviewed used objective instrumented outcome measures, and the measures applied in these studies used mainly summary statistics like the RMS of the amplitude of COP recordings. Many of these measures appeared unable to detect small changes in postural control, some of which actually were observed using clinical measures, as showed in Figure 1, Figure 2 and Table $1[24,33,38,43,44]$. Using summary statistics may mask variability in postural sway patterns. There is a growing recognition that the time-varying structure of postural sway patterns contains valuable information on healthy and pathologic motor control [18,80-84]. To reveal these sway patterns, different types of data analysis techniques are necessary. A time-dependent analysis of variability and stability using measures derived from the theory of stochastic dynamics is a relatively new approach to quantify postural variability and stability [18,80-84]. Nowadays a wide variety of methods for quantifying balance ability using sway patterns has been applied and shown sensitive to detect change due to aging and pathologies including stabilogram diffusion plots (SDP) [81], recurrence quantification analysis (RQA) [85], detrended fluctuation analysis (DFA) $[83,86]$, sample entropy (SEn) [87], Lyapunov exponent ( $\lambda \max )$ [88] and Rambling and trembling analysis [89]. The new methods assess fluctuations in postural control signals and are suggested to be associated with the neuromuscular mechanisms underlying postural control [81]. These measures, based on the time-varying structure of postural sway patterns, are not depending on a specific sensor, but can be applied on various data sources including COP and acceleration data. Unlike clinical measures no healthcare professional is needed to guide the measurement and no ceiling effects are observed. However, the optimal selection of discriminative sway parameters is under continuous dispute; no gold standard is present for the quantification of postural control. In addition to the information provided about postural control, key aspects that remain to be examined in this respect are the accuracy, sensitivity and reliability of outcome measures. Internal measures hold the advantage that they are based on data recorded over a timeframe of hours, days or even weeks, since data is acquired constantly during gameplay, whereas external measures rely on short balance tests independent from the game environment. Internal measures on the other hand are calculated using sensor systems that need to be affordable for the individual user, thus compromising on accuracy of equipment is inevitable and the quality of measurements is usually lower than lab equipment used for external measures. However, as high quality sensors rapidly become smaller and more affordable, internal outcome measures can be based on data recorded with high precision and the challenge will focus on finding outcome measures that are reliable and sensitive to change in balance control of community dwelling elderly playing exergames.

Recent developments in sensor technology, game design and cheap processing power offers the unique possibility to calculate the above mentioned type of instrumented outcome measures during training activity. This clears the road for several breakthroughs in balance assessment and training. First, balance can be measured while playing exergames, leaving the user unaware of the measurements with no involvement of a healthcare professional or need for measurements in a laboratory setting. Second, the measurements allow for analysis over a variety of timeframes; balance can be quantified in the order of minutes and can 
serve as direct input in gameplay but results can also be stored and compared with measurements over months or even years. This allows personalized training programs and feedback which has shown to increase learning rate [90]. Moreover, measurements can be compared with peers using telemonitoring principles.

An additional advantage of exergames is motivation, although it is hardly examined quantitatively and compared with control treatment. The reviewed studies showed that exergame intervention groups found the training more appealing than traditional exercises [23], were more motivated to exercise [24] and showed better improvements than controls on clinical outcome measures $[29,34,40,42]$.

We propose to utilize the latest advances in sensor technology and develop algorithms to analyze multivariate time series of postural control over different timescales, for assessment of postural control both during gameplay as well as after gameplay, so to derive specific information regarding the postural control of the user and thereby adapting the exergame training program most efficiently to the individual user. Future developments should focus on developing algorithms that convert sensor data into information regarding postural control.

\section{Conclusion}

Exergames have shown to hold interesting opportunities for improving balance ability in older adults. Although the number of controlled studies examining exergames remains small, the included studies report high enjoyment and motivation to perform exercises and several studies indeed showed an increase in balance ability using clinical and instrumented outcome measures after the training period. Current balance outcome measures however contain some caveats. We propose to utilize the recent advances in sensor technology and data analysis algorithms for quantification of balance ability during exergame training sessions, thereby unlocking new possibilities that exergames encompass for improving balance ability in community dwelling elderly.

\section{Competing interests}

The authors declare that they have no competing interests.

\section{Authors' contributions}

MvD was involved in the conception of the review, the search and analysis and interpretation of the studies, and the writing of the manuscript. CJCL was involved in the conception of the review, interpretation and writing of the manuscript. JS, KP, and GJV were involved in revising of the manuscript. All authors read and approved the final manuscript.

\section{Acknowledgements}

This work was supported by SPRINT research center, the Province of Drenthe, the Municipality of Assen, the European Fund for Regional Development and the Ministry of Economic Affairs, Peaks in the Delta.

\section{Author details}

${ }^{1}$ INCAS $^{3}$, Dr. Nassaulaan 9, 9401 HJ Assen, The Netherlands. ${ }^{2}$ Center for Human Movement Sciences, University of Groningen, University Medical Center Groningen, Hanzeplein 1, 9713 GZ Groningen, The Netherlands.

${ }^{3}$ Center for Rehabilitation, University Medical Center Groningen, University of Groningen, Hanzeplein 1, 9713 GZ Groningen, The Netherlands. " Department of Biomechanical Engineering, University of Twente, P.O. Box 217, 7500 AE Enschede, The Netherlands.

Received: 21 August 2012 Accepted: 19 September 2013

Published: 25 September 2013

\section{References}

1. Tinetti ME, Speechley M, Ginter SF: Risk factors for falls among elderly persons living in the community. N Engl J Med 1988, 319:1701-1707.

2. Campbell AJ, Borrie MJ, Spears GF: Risk factors for falls in a communitybased prospective study of people 70 years and older. J Gerontol 1989, 44:M112-M117.

3. Sterling DA, O'Connor JA, Bonadies J: Geriatric falls: injury severity is high and disproportionate to mechanism. J Trauma 2001, 50:116-119.

4. Rubenstein LZ: Falls in older people: epidemiology, risk factors and strategies for prevention. Age Ageing 2006, 35(Suppl 2):ii37-ii41.

5. Tinetti ME, Williams CS: Falls, injuries due to falls, and the risk of admission to a nursing home. N Engl J Med 1997, 337:1279-1284.

6. Delbaere K, Close JCT, Heim J, Sachdev PS, Brodaty H, Slavin MJ, Kochan NA, Lord SR: A multifactorial approach to understanding fall risk in older people. J Am Geriatr Soc 2010, 58:1679-1685.

7. Lajoie Y, Gallagher SP: Predicting falls within the elderly community: comparison of postural sway, reaction time, the Berg balance scale and the Activities-specific Balance Confidence ( $A B C)$ scale for comparing fallers and non-fallers. Arch Gerontol Geriatr 2004, 38:11-26.

8. Pollock AS, Durward BR, Rowe PJ, Paul JP: What is balance? Clin Rehabil 2000, 14:402-406.

9. Laughton CA, Slavin M, Katdare K, Nolan L, Bean JF, Kerrigan DC, Phillips E, Lipsitz LA, Collins JJ: Aging, muscle activity, and balance control: physiologic changes associated with balance impairment. Gait Posture 2003, 18:101-108.

10. Horak FB, Shupert CL, Mirka A: Components of postural dyscontrol in the elderly: a review. Neurobiol Aging 1989, 10:727-738.

11. Woollacott M, Shumway-Cook A: Attention and the control of posture and gait: a review of an emerging area of research. Gait \& posture 2002, 16:1-14.

12. Beauchet $O$, Dubost V, Herrmann FR, Kressig RW: Stride-to-stride variability while backward counting among healthy young adults. J Neuroeng Rehabil 2005, 2:26.

13. Lajoie Y, Teasdale N, Bard C, Fleury M: Attentional demands for static and dynamic equilibrium. Exp Brain Res 1993, 97:139-144.

14. Brown LA, Shumway-Cook A, Woollacott MH: Attentional demands and postural recovery: the effects of aging. J Gerontol A Biol Sci Med Sci 1999, 54:165-171.

15. Maylor EA, Wing AM: Age differences in postural stability are increased by additional cognitive demands. J Gerontol B Psychol Sci Soc Sci 1996, 51:143-154.

16. Teasdale N, Simoneau M: Attentional demands for postural control: the effects of aging and sensory reintegration. Gait \& posture 2001, $14: 203-210$.

17. Ijmker T, Lamoth CJC: Gait and cognition: the relationship between gait stability and variability with executive function in persons with and without dementia. Gait \& posture 2012, 35:126-130.

18. Lamoth CJ, van Deudekom FJ, van Campen JP, van Campen JP, van Appels BA, de Pijnappels M: Gait stability and variability measures show effects of impaired cognition and dual tasking in frail people. J Neuroeng Rehabil 2011, 8:2.

19. Kannus $\mathrm{P}$, Sievänen $H$, Palvanen $M$, Järvinen T, Parkkari J: Prevention of falls and consequent injuries in elderly people. Lancet 2005, 366:1885-1893.

20. Doughty $K$, Lewis $R$, Mclntosh A: The design of a practical and reliable fall detector for community and institutional telecare. J Telemed Telecare 2000, 6(Suppl 1):S150-S154.

21. Gillespie LD, Robertson MC, Gillespie WJ, Lamb SE, Gates S, Cumming RG, Rowe $\mathrm{BH}$ : Interventions for preventing falls in older people living in the community. Cochrane Database Syst Rev 2009, 2:CD007146. 
22. Sherrington C, Whitney JC, Lord SR, Herbert RD, Cumming RG, Close JCT: Effective exercise for the prevention of falls: a systematic review and meta-analysis. J Am Geriatr Soc 2008, 56:2234-2243.

23. Betker A, Szturm T, Moussavi Z: Development of an interactive motivating tool for rehabilitation movements. Conf Proc IEEE Eng Med Biol Soc 2005, $3: 2341-2344$.

24. Fitzgerald D, Trakarnratanakul N, Smyth B, Caulfield B: Effects of a wobble board-based therapeutic exergaming system for balance training on dynamic postural stability and intrinsic motivation levels. J Orthop Sports Phys Ther 2010, 40:11-19.

25. Lamoth CJC, Caljouw SR, Postema K: Active video gaming to improve balance in the elderly. Stud Health Technol Inform 2011, 167:159-164.

26. Lamoth CJC, Alingh R, Caljouw S: Exergaming for elderly: Effects of different types of game feedback on performance of a balance task. Stud Health Technol Inform 2012. in press.

27. Plow MA, McDaniel C, Linder S, Alberts JL: A Scoping Review of Exergaming for Adults with Systemic Disabling Conditions. Journal of Bioengineering and Biomedical Sciences 2011, 01:1-11.

28. Rademaker $\mathrm{A}$, van der Linden S, Wiersinga J: SilverFit, a virtual rehabilitation system. Gerontechnology 2009, 8:119.

29. Gil-Gómez J-A, Lloréns R, Alcañiz M, Colomer C: Effectiveness of a Wii balance board-based system (eBaViR) for balance rehabilitation: a pilot randomized clinical trial in patients with acquired brain injury. J Neuroeng Rehabil 2011, 8:30.

30. Studenski S, Perera S, Hile E, Keller V, Spadola-Bogard J, Garcia J: Interactive video dance games for healthy older adults. J Nutr Health Aging 2010, 14:850-852.

31. Smith ST, Sherrington C, Studenski S, Schoene D, Lord SR: A novel Dance Dance Revolution (DDR) system for in-home training of stepping ability: basic parameters of system use by older adults. Br J Sports Med 2011, 45:441-445

32. Yavuzer G, Senel A, Atay MB, Stam HJ: Playstation eyetoy games" improve upper extremity-related motor functioning in subacute stroke: a randomized controlled clinical trial. Eur J Phys Rehabil Med 2008, 44:237-244.

33. Kosse N, Caljouw S, Vuijk P, CJC L: Exergaming: interactive balance training in healthy community-dwelling elderly. Journal of Cyber Therapy \& Rehabilitation 2011, 4:399-407.

34. Saposnik G, Teasell R, Mamdani M, Hall J, Mcllroy W, Cheung D, Thorpe KE, Cohen LG, Bayley M: Effectiveness of Virtual Reality Using Wii Gaming Technology in Stroke Rehabilitation. A Pilot Randomized Clinical Trial and Proof of Principle. Stroke 2010, 41:1477-1484.

35. Analog Devices Inc: Small, Low Power, 3-axis \pm 3 g iMEMS ${ }^{\circledR}$ Accelerometer. Norwood: Analog Devices Inc; 2007

36. Fitzgerald D, Trakarnratanakul N, Dunne L, Smyth B, Caulfield B: Development and user evaluation of a virtual rehabilitation system for wobble board balance training. In Annual International Conference of the IEEE Engineering in Medicine and Biology Society. IEEE Engineering in Medicine and Biology Society; 2008:4194-4198.

37. Billis AS, Konstantinidis El, Mouzakidis C, Tsolaki MN, Pappas C, Bamidis PD: A Game-Like Interface for Training Seniors ' Dynamic Balance and Coordination. IFMBE Proc 2010, 29:691-694.

38. Bisson E, Contant B, Sveistrup H, Lajoie Y: Functional balance and dual-task reaction times in older adults are improved by virtual reality and biofeedback training. Cyberpsychol Behav 2007, 10:16-23.

39. Lange B, Flynn S, Proffitt R, Chang C-Y, Rizzo AS: Development of an interactive game-based rehabilitation tool for dynamic balance training Top Stroke Rehabil 2010, 17:345-352.

40. Lange B, Flynn S, Chang C, Liang W, Chieng C, Si YCN, Rizzo A: Development of an interactive stepping game to reduce falls in older adults. In 8th Intl Conf. Disability, Virtual Reality and Associated Technologies. International Society for Virtual Rehabilitation; 2010:223-228.

41. Nitz JC, Kuys S, Isles R, Fu S: Is the Wii Fit a new-generation tool for improving balance, health and well-being? A pilot study. Climacteric 2010, 13:487-491.

42. Williams MA, Soiza RL, Jenkinson AM, Stewart A: EXercising with Computers in Later Life (EXCELL) - pilot and feasibility study of the acceptability of the Nintendo ${ }^{\oplus}$ WiiFit in community-dwelling fallers. BMC research notes 2010, 3:238.

43. Young W, Ferguson S, Brault S, Craig C: Assessing and training standing balance in older adults: A novel approach using the "Nintendo Wii" Balance Board. Gait \& posture 2010, 33:303-305.
44. Heiden E, Lajoie Y: Games-based biofeedback training and the attentional demands of balance in older adults. Aging Clin Exp Res 2009, 22:367-373.

45. Deutsch JE, Robbins D, Morrison J, Guarrera Bowlby P: Wii-based compared to standard of care balance and mobility rehabilitation for two individuals post-stroke. In Virtual Rehabilitation International Conference. IEEE; 2009:117-120.

46. Flynn S, Palma P, Bender A: Feasibility of using the Sony PlayStation 2 gaming platform for an individual poststroke: a case report. J Neurol Phys Ther 2007, 31:180-189.

47. Lange B, Flynn S, Rizzo A: Initial usability assessment of off-the-shelf video game consoles for clinical game-based motor rehabilitation. Phys Ther Rev 2009, 14:355-363.

48. Rand D, Kizony R, Weiss PTL: The Sony PlayStation II EyeToy: low-cost virtual reality for use in rehabilitation. J Neurol Phys Ther 2008, 32:155-163.

49. Khoshelham K, Elberink SO: Accuracy and Resolution of Kinect Depth Data for Indoor Mapping Applications. Sensors 2012, 12:1437-1454.

50. Berg KO, Wood-Dauphinee SL, Williams Jl, Maki B: Measuring balance in the elderly: validation of an instrument. Can J Public Health 1992, 83:S7-S11.

51. Podsiadlo D, Richardson S: The timed "Up \& Go": a test of basic functional mobility for frail elderly persons. J Am Geriatr Soc 1991, 39:142-148.

52. Steffen TM, Hacker TA, Mollinger L: Age- and gender-related test performance in community-dwelling elderly people: Six-Minute Walk Test, Berg Balance Scale, Timed Up \& Go Test, and gait speeds. Physical therapy 2002, 82:128-137.

53. Tyson SF, DeSouza LH: Development of the Brunel Balance Assessment: a new measure of balance disability post stroke. Clin Rehabil 2004, 18:801-810.

54. Duncan PW, Weiner DK, Chandler J, Studenski S: Functional reach: a new clinical measure of balance. J Gerontol 1990, 45:M192-M197.

55. Perron M, Malouin F, Moffet $\mathrm{H}$ : Assessing advanced locomotor recovery after total hip arthroplasty with the timed stair test. Clin Rehabil 2003, 17:780-786

56. Hill KD: A New Test of Dynamic Standing Balance for Stroke Patients: Reliability, Validity and Comparison with Healthy Elderly. Physiother Can 1996, 48:257-262.

57. McDowell BC, Kerr C, Parkes J, Cosgrove A: Validity of a 1 minute walk test for children with cerebral palsy. Dev Med Child Neurol 2005, 47:744-748.

58. Van Hedel HJ, Wirz M, Dietz V: Assessing walking ability in subjects with spinal cord injury: validity and reliability of 3 walking tests. Arch Phys Med Rehabil 2005, 86:190-196

59. O'Shea SD, Taylor NF, Paratz JD: Measuring muscle strength for people with chronic obstructive pulmonary disease: retest reliability of handheld dynamometry. Arch Phys Med Rehabil 2007, 88:32-36.

60. Howe JA, Inness EL, Venturini A, Williams JI, Verrier MC: The Community Balance and Mobility Scale - a balance measure for individuals with traumatic brain injury. Clin Rehabil 2006, 20:885-895.

61. Kinzey SJ, Armstrong CW: The reliability of the star-excursion test in assessing dynamic balance. J Orthop Sports Phys Ther 1998, 27:356-360.

62. Hess RJ, Brach JS, Piva SR, VanSwearingen JM: Walking skill can be assessed in older adults: validity of the Figure-of-8 Walk Test. Phys Ther 2010, 90:89-99.

63. Rossiter-Fornoff JE, Wolf SL, Wolfson LI, Buchner DM: A cross-sectional validation study of the FICSIT common data base static balance measures. J Gerontol A Biol Sci Med Sci 1995, 50:291-297.

64. Cimolin V, Galli M, Rigoldi C, Grugni G, Vismara L, Mainardi L, Capodaglio P. Fractal dimension approach in postural control of subjects with PraderWilli Syndrome. J Neuroeng Rehabil 2011, 8:45.

65. Galli M, Cimolin V, Vismara L, Grugni G, Camerota F, Celletti C, Albertini G, Rigoldi $C$, Capodaglio P: The effects of muscle hypotonia and weakness on balance: a study on Prader-Willi and Ehlers-Danlos syndrome patients. Res Dev Disabil 2011, 32:1117-1121.

66. Baratto L, Morasso PG, Re C, Spada G: A new look at posturographic analysis in the clinical context: sway-density versus other parameterization techniques. Motor control 2002, 6:246-270.

67. Menegoni F, Tacchini E, Bigoni M, Vismara L, Priano L, Galli M, Capodaglio P. Mechanisms underlying center of pressure displacements in obese subjects during quiet stance. J Neuroeng Rehabil 2011, 8:20.

68. Wikstrom EA, Tillman MD, Smith AN, Borsa PA: A new force-plate technology measure of dynamic postural stability: the dynamic postural stability index. J Athl Train 2005, 40:305-309.

69. Wechsler D: Wechsler Adult Intelligence Scale-Revised. New York: Psychological Corporation; 1981. 
70. Ware JE, Sherbourne CD: The MOS 36-item short-form health survey (SF-36). I. Conceptual framework and item selection. Med Care 1992, 30:473-483.

71. Tinetti ME, Richman D, Powell L: Falls efficacy as a measure of fear of falling. J Gerontol 1990, 45:P239-P243.

72. Yardley L, Beyer N, Hauer K, Kempen G, Piot-Ziegler C, Todd C: Development and initial validation of the Falls Efficacy ScaleInternational (FES-I). Age ageing 2005, 34:614-619.

73. Powell LE, Myers AM: The Activities-specific Balance Confidence (ABC) Scale. J Gerontol A Biol Sci Med Sci 1995, 50:28-34.

74. Schoene D, Lord SR, Verhoef P, Smith ST: A novel video game-based device for measuring stepping performance and fall risk in older people. Arch Phys Med Rehabil 2011, 92:947-953.

75. Agmon M, Perry CK, Phelan E, Demiris G, Nguyen HQ: A pilot study of wii fit exergames to improve balance in older adults. J Geriatr Phys Ther 2011, 34:161-167.

76. Lange B, Koenig S, Chang C-Y, McConnell E, Suma E, Bolas M, Rizzo A: Designing informed game-based rehabilitation tasks leveraging advances in virtual reality. Disabil Rehabil 2012, 34:1863-1870.

77. Patel $\mathrm{S}$, Park $\mathrm{H}$, Bonato $\mathrm{P}$, Chan $\mathrm{L}$, Rodgers $\mathrm{M}$ : A review of wearable sensors and systems with application in rehabilitation. J Neuroeng Rehabil 2012, 9:21.

78. Miao P, Mitcheson PD, Holmes a S, Yeatman EM, Green TC, Stark BH: Mems inertial power generators for biomedical applications. Microsyst Technol 2006, 12:1079-1083.

79. Blum L, Korner-Bitensky N: Usefulness of the Berg Balance Scale in stroke rehabilitation: a systematic review. Phys Ther 2008, 88:559-566.

80. Daffertshofer A, Lamoth CJC, Meijer OG, Beek PJ: PCA in studying coordination and variability: a tutorial. Clin Biomech 2004, 19:415-428.

81. Collins JJ, De Luca CJ: Open-loop and closed-loop control of posture: a random-walk analysis of center-of-pressure trajectories. Exp Brain Res 1993, 95:308-318.

82. Lipsitz LA, Cupples LA, Kiely DK, Peng CK, Goldberger AL, Starobinets OV Priplata AA, Costa MD, Kang HG: Frailty and the degradation of complex balance dynamics during a dual-task protocol. I Gerontol A Biol Sci Med Sci 2009, 64:1304-1311.

83. Lamoth CJC, van Lummel RC, Beek PJ: Athletic skill level is reflected in body sway: a test case for accelometry in combination with stochastic dynamics. Gait \& posture 2009, 29:546-551.

84. Lamoth CJC, van Heuvelen MJG: Sports activities are reflected in the local stability and regularity of body sway: Older ice-skaters have better postural control than inactive elderly. Gait \& posture 2011, 35:489-493.

85. Riley MA, Balasubramaniam R, Turvey MT: Recurrence quantification analysis of postural fluctuations. Gait \& posture 1999, 9:65-78.

86. Peng CK, Havlin S, Stanley HE, Goldberger AL: Quantification of scaling exponents and crossover phenomena in nonstationary heartbeat time series. Chaos (Woodbury, N.Y.) 1995, 5:82-87.

87. Richman JS, Moorman JR: Physiological time-series analysis using approximate entropy and sample entropy. Am J Physiol Heart Circ Physiol 2000, 278:H2039-H2049.

88. Rosenstein M, Collins J, De Luca C: A practical method for calculating largest Lyapunov exponents from small data sets. Physica $D$-nonlinear phenomena 1993, 65:117-134.

89. Zatsiorsky VM, Duarte M: Instant equilibrium point and its migration in standing tasks: rambling and trembling components of the stabilogram. Motor control 1999, 3:28-38.

90. Bilodeau E: Motor-skills learning. Annu Rev Psychol 1961, 12:243-280.

doi:10.1186/1743-0003-10-101

Cite this article as: van Diest et al:: Exergaming for balance training of elderly: state of the art and future developments. Journal of NeuroEngineering and Rehabilitation 2013 10:101.

\section{Submit your next manuscript to BioMed Central and take full advantage of:}

- Convenient online submission

- Thorough peer review

- No space constraints or color figure charges

- Immediate publication on acceptance

- Inclusion in PubMed, CAS, Scopus and Google Scholar

- Research which is freely available for redistribution

Submit your manuscript at www.biomedcentral.com/submit
Ciomed Central 